CrossMark <click for updates

Cite this: Phys. Chem. Chem. Phys., 2016, 18, 15133

Received 25th March 2016,

Accepted 6th May 2016

DOI: $10.1039 / c 6 c p 01998 f$

www.rsc.org/pccp

\title{
Electronic state-lifetime interference in resonant Auger spectra: a tool to disentangle overlapping core-excited states
}

\author{
Gildas Goldsztejn, ${ }^{\text {a }}$ Tatiana Marchenko, ${ }^{a b}$ Denis Céolin, ${ }^{b}$ Loïc Journel, ${ }^{a b}$ \\ Renaud Guillemin, ${ }^{\mathrm{ab}}$ Jean-Pascal Rueff, ${ }^{\text {ab }}$ Rajesh K. Kushawaha, ${ }^{a}$ Ralph Püttner, ${ }^{c}$ \\ Maria Novella Piancastelli ${ }^{\text {ad }}$ and Marc Simon ${ }^{\text {ab }}$
}

\begin{abstract}
We have measured resonant-Auger decay following $\mathrm{Cl}_{1} \mathrm{~s}^{-1}$ excitations in $\mathrm{HCl}$ and $\mathrm{CH}_{3} \mathrm{Cl}$ molecules, and extracted the pseudo-cross sections of different $\mathrm{Cl} 2 \mathrm{p}^{-2}$ final states. These cross sections show clear evidence of shake processes as well as contributions of electronic state-lifetime interference (ELI). To describe the spectra we developed a fit approach that takes into account ELI contributions and ultrafast nuclear dynamics in dissociative core-excited states. Using this approach we utilized the ELI contributions to obtain the intensity ratios of the overlapping states $\mathrm{Cl} 1 \mathrm{~s}^{-1} 4 \mathrm{p} \pi / 1 \mathrm{~s}^{-1} 4 \mathrm{p \sigma}$ in $\mathrm{HCl}$ and $\mathrm{Cl}$ $1 \mathrm{~s}^{-1} 4 \mathrm{pe} / 1 \mathrm{~s}^{-1} 4 \mathrm{pa}_{1}$ in $\mathrm{CH}_{3} \mathrm{Cl}$. The experimental value for $\mathrm{HCl}$ is compared with theoretical results showing satisfactory agreement.
\end{abstract}

\section{Introduction}

Ultrafast dynamics following core excitation in small isolated molecules has been extensively studied since the mid 80 s in the soft X-ray energy range. The main experimental means to probe such phenomena is to study non-radiative decay which is the dominant decay path for light elements, i.e. the Auger electrons emitted after resonant excitation from a core shell. In the soft $\mathrm{X}$-ray range, the lifetime of the intermediate state created is in the order of a few femtoseconds. During this time the nuclear wavepacket propagates on the potential energy surfaces (PES) of the excited states and may lead, in the case of dissociative potentials, to breaking of the molecular bond prior to electronic relaxation. A large body of work has evidenced competition between molecular and atomic de-excitations on different molecules such as $\mathrm{HBr},{ }^{1} \mathrm{HCl},{ }^{2-7} \mathrm{H}_{2} \mathrm{O},{ }^{8} \mathrm{O}_{2}{ }^{9}$ and $\mathrm{NH}_{3} \cdot{ }^{10}$

Recently the use of third-generation synchrotrons and extensive theoretical work on radiative decays ${ }^{11}$ have also allowed studying nuclear dynamics from resonant inelastic X-ray scattering (RIXS) measurements in the hard X-ray energy range $(>1 \mathrm{keV})^{12,13}$ which

\footnotetext{
${ }^{a}$ Sorbonne Universités, UPMC Univ Paris 06, CNRS, UMR 7614,

Laboratoire de Chimie Physique-Matière et Rayonnement, F-75005, Paris, France. E-mail: gildas.goldsztejn@upmc.fr

${ }^{b}$ Synchrotron SOLEIL, l'Orme des Merisiers, Saint-Aubin, BP 48, F-91192 Gif-sur-Yvette Cedex, France

${ }^{c}$ Institut für Experimentalphysik, Freie Universität Berlin, Arnimallee 14, D-14195 Berlin, Germany

${ }^{d}$ Department of Physics and Astronomy, Uppsala University, P. O. Box 516,

SE-751 20 Uppsala, Sweden
}

gives access to shorter lifetimes of intermediate states, i.e. 1 femtosecond or less. Complete dissociation does not occur on such a short timescale; nevertheless, one can observe elongation of the chemical bond. ${ }^{12-15}$ Some of the measurements in $\mathrm{CH}_{3} \mathrm{Cl}$ presented in this paper have been discussed in detail by Piancastelli et al. ${ }^{14}$ in particular those concerning nuclear dynamics upon excitation of $\mathrm{Cl} 1 \mathrm{~s} \rightarrow$ Lowest Unoccupied Molecular Orbital (LUMO).

In the hard X-ray range, the different intermediate states overlap quite strongly due to large lifetime broadening. They can therefore be coherently excited, meaning that the excitation by the incident photon creates several intermediate states that can either decay in the same final state or in different ones. Fig. 1 is a schematic representation of this description, in which we, firstly, represented by a vertical arrow the coherent excitation of two different intermediate states: $1 \mathrm{~s}^{-1}$ core-hole with one electron promoted to the LUMO, which in this case is dissociative, and $1 \mathrm{~s}^{-1}$ Rydberg, which is a bound state. Secondly, the electronic relaxation of the core-hole state, in which the excited electron can remain in the same orbital is also represented. This process is called the spectator Auger decay and is represented by gray arrows in Fig. 1. There is also a possibility for the excited electron to be promoted to another orbital during the relaxation process. The dashed black line represents a "shakedown" from a Rydberg orbital to the LUMO, while the dotted black line represents a "shake-up" from the LUMO to the Rydberg orbital.

When different overlapping intermediate states of the same symmetry are coherently excited and decay to the same final 


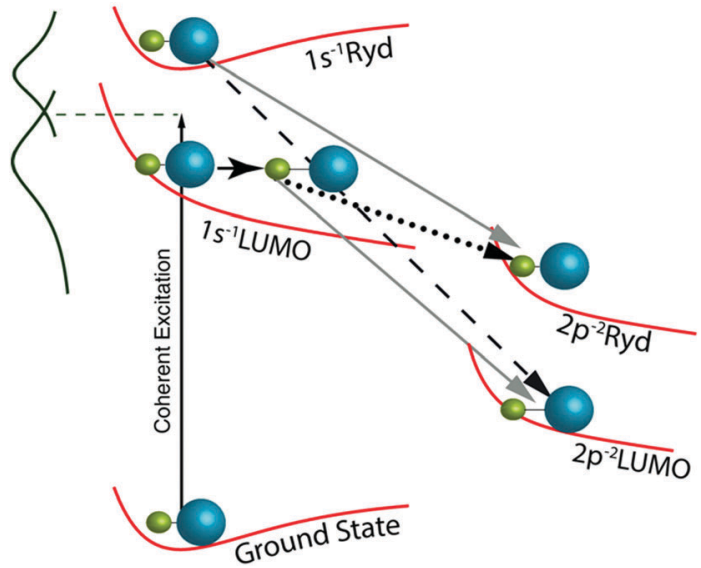

Fig. 1 Schematic representation of ELI in the case of bound and dissociative intermediate states. The vertical arrow represents the coherent excitation of two different intermediate states: the dissociative $1 \mathrm{~s}^{-1}$ LUMO excitation in which the chemical bond elongates, and a bound $1 \mathrm{~s}^{-1}$ Ryd excitation. The molecule can decay by a spectator Auger process (grey arrows) or by shake processes: a shake-up from $1 \mathrm{~s}^{-1}$ LUMO to $2 \mathrm{p}^{-2}$ Ryd final state (dotted black line) or a shake-down from the $1 \mathrm{~s}^{-1}$ Ryd to $2 \mathrm{p}^{-2}$ LUMO final state (dashed black line). ELI is caused by the fact that several intermediate states decay into a same final state.

state, electronic state-lifetime interference (ELI) may occur. This manifests itself in the line profiles and energy positions of the peak maxima in the partial cross sections of the different $\mathrm{Cl} 2 \mathrm{p}^{-2}$ final states. However, the present set-up does not allow performing experiments at the magic angle (see Section 2) so that we can only compare relative intensities of the different contributions; thus we prefer to use throughout this publication the term of pseudo-cross section rather than partial cross sections.

While the influence of ELI on the energy positions is in some cases clearly visible, see e.g. $\mathrm{HCl}$ below, its influence on the line profile of the pseudo-cross sections is subtler and requires detailed analysis. Note that, for dissociative intermediate states, nuclear dynamics occurring in the excited state has to be taken into account.

ELI was theoretically predicted long ago, since Fano demonstrated interference between discrete and continuum states. ${ }^{16}$ Åberg developed a unified theory for Auger electron emission taking into account interference terms (both vibrational and electronic ${ }^{17}$ and Cesar et al. elaborated a theoretical description of this phenomenon using scattering theory in both non-radiative and radiative decays. ${ }^{18}$ However, only few experimental evidences of ELI can be found in the literature. Levin et al. performed coincidence measurements after a K-shell excitation in argon, ${ }^{19}$ Rubensson et al. performed measurements at the $\mathrm{K}$ threshold in the neon atom ${ }^{20}$ and LeBrun et al. studied radiationless resonant scattering in argon. ${ }^{21}$ In all these experimental papers, limited experimental resolution did not allow disentangling the contributions of the intermediate states to one particular final state.

Recently, ELI has been observed in argon excited at the K-edge, using the HArd X-ray PhotoEmission Spectroscopy (HAXPES) set-up at the GALAXIES beamline. ${ }^{22}$ The results obtained are in good agreement with the theoretical predictions by Armen et al. ${ }^{23}$
Another evidence of ELI in atoms has been demonstrated using HAXPES at the L-edges of xenon. ${ }^{24}$ There are even less experimental evidences of ELI in molecules. Kavčič et al. showed a proof of this interference in $\mathrm{HCl}$ using RIXS; ${ }^{25}$ however, due to the stricter selection rules for radiative decays than for non-radiative decays, the observed interference effect was relatively weak.

This work presents the resonant Auger spectra for the molecules $\mathrm{HCl}$ and $\mathrm{CH}_{3} \mathrm{Cl}$ measured at the $\mathrm{Cl}$ K-edge and displayed as 2D-maps recorded at different photon energies tuned with a small step around the $\mathrm{Cl} 1 \mathrm{~s} \rightarrow$ LUMO resonance and further beyond the ionization threshold. From these spectra the pseudo-cross sections for the different $\mathrm{Cl} 2 \mathrm{p}^{-2}$ final states are derived. For $\mathrm{HCl}$, the pseudo-cross sections show strong contributions of ELI in terms of energy shifts of the pseudocross section maxima. For $\mathrm{CH}_{3} \mathrm{Cl}$, the ELI contributions are more subtle and can be pinpointed only by simulations taking into account nuclear dynamics. Importantly, we show that the ELI contributions can be utilized to disentangle unresolved states in the absorption spectrum, more precisely: $\mathrm{Cl} 1 \mathrm{~s}^{-1} 4 \mathrm{p}$ intermediate states of different symmetries, namely $\mathrm{Cl}_{1 \mathrm{~s}^{-1} 4 \mathrm{p} \sigma /}$ $4 \mathrm{p} \pi$ in $\mathrm{HCl}$ and $1 \mathrm{~s}^{-1} 4 \mathrm{pe} / 4 \mathrm{pa}_{1}$ in $\mathrm{CH}_{3} \mathrm{Cl}$. Due to this finding the present experimental approach becomes complementary to symmetry-resolved X-ray absorption spectroscopy, see the review of Adachi et al. ${ }^{26}$ and references therein. In the latter method the disentanglement of the overlapping states with different symmetries is more direct; however, the method is limited to linear molecules and has not been applied to spectra in the hard $\mathrm{X}$-ray regime yet.

Our article is organized as follows: Section 2 gives a description of the experimental setup and the Auger measurements in $\mathrm{HCl}$ and $\mathrm{CH}_{3} \mathrm{Cl}$. Section 3 provides details of the formalism of our simulations. Our analysis is presented in Section 4 and we give our conclusions in Section 5.

\section{Experimental set-up and results}

The measurements presented in this article were performed at the GALAXIES beamline of the French national synchrotron SOLEIL. ${ }^{27}$ This beamline is optimized for an energy range of 2.3-12 keV and has an end-station dedicated to HAXPES. A complete description of the HAXPES end-station can be found in Céolin et al. ${ }^{28}$ Briefly, the linearly polarized light comes from a U20 undulator, and is monochromatized by a Si(111) doublecrystal set-up. Moreover, the beamline is equipped with a collimating mirror of two different coatings, used for different energy regions, and one toroidal mirror to focus the beam onto the end-station. A hemispherical analyzer EW4000 Scienta is mounted in order to analyze electrons with a large acceptance angle. The lens axis of the analyzer is set parallel to the polarization of the synchrotron radiation. The analyzer resolution was set to $200 \mathrm{meV}$ for the $\mathrm{HCl}$ measurement and to $150 \mathrm{meV}$ for $\mathrm{CH}_{3} \mathrm{Cl}$. The photon bandwidth has been represented by a Gaussian function of $400 \mathrm{meV}$ full width at half maximum (FWHM) and the Doppler broadening was estimated to be $100 \mathrm{meV}$ by assuming that the gas cell is at 
room temperature. Based on these values, the total instrumental resolution results in $460 \mathrm{meV}$ for $\mathrm{HCl}$ and $440 \mathrm{meV}$ for $\mathrm{CH}_{3} \mathrm{Cl}$. The unique performances of the HAXPES end-station have allowed evidencing fine phenomena such as atomic Doppler effect ${ }^{29}$ or double core-hole simultaneous excitation and ionization. ${ }^{30}$

The process of KLL Auger emission after resonant $\mathrm{Cl} 1 \mathrm{~s}$ excitation can be written as

$$
\gamma+\mathrm{Cl} \rightarrow \mathrm{Cl}^{*}\left(1 \mathrm{~s}^{-1} \mathrm{~V}^{\prime}\right) \rightarrow \mathrm{Cl}^{+}\left(2 \mathrm{p}^{-2} \mathrm{~V}\right)+\mathrm{e}_{\mathrm{KLL}}^{-}
$$

In the following $\mathrm{V}$ can either represent the LUMO, which is the $6 \sigma$ orbital for $\mathrm{HCl}$ and the $8 \mathrm{a}_{1}$ orbital for $\mathrm{CH}_{3} \mathrm{Cl}$, or a Rydberg orbital. Moreover, the prime describes an orbital in the presence of a Cl $1 \mathrm{~s}^{-1}$ core hole while the orbitals without prime are in the presence of a $\mathrm{Cl} 2 \mathrm{p}^{-2}$ double core hole. As a consequence orbitals indicated by $\mathrm{V}$ and $\mathrm{V}^{\prime}$ are not orthogonal to each other, see below. $\mathrm{e}_{\mathrm{KLL}}{ }^{-}$is the resonant KLL Auger electron emitted during the decay of the $\mathrm{K}$ hole and $\gamma$ is the incoming photon. In Fig. 2 we show the resonant $\mathrm{KLL}$ Auger decay of $\mathrm{HCl}$ and $\mathrm{CH}_{3} \mathrm{Cl}$ as $2 \mathrm{D}$-maps. The $2 \mathrm{D}$-maps represent the electron kinetic energy as a function of the incident photon energy and the intensity is represented by a colorscale. These measurements were carried out in the kinetic energy range $2350-2400 \mathrm{eV}$ with a $0.05 \mathrm{eV}$ step for $\mathrm{HCl}$ and $0.1 \mathrm{eV}$ step for $\mathrm{CH}_{3} \mathrm{Cl}$. The incident photon energy was varied from 2820 to $2832 \mathrm{eV}$ using steps of $0.1 \mathrm{eV}$ for $\mathrm{HCl}$ and $0.2 \mathrm{eV}$ for $\mathrm{CH}_{3} \mathrm{Cl}$. The partial electron yield (PEY) spectra, obtained by integrating the 2D-maps along the kinetic-energy axis, are plotted in the upper part of Fig. 2 as a visual aid to identify the resonances of the intermediate states; for detailed assignment of these resonances see below.

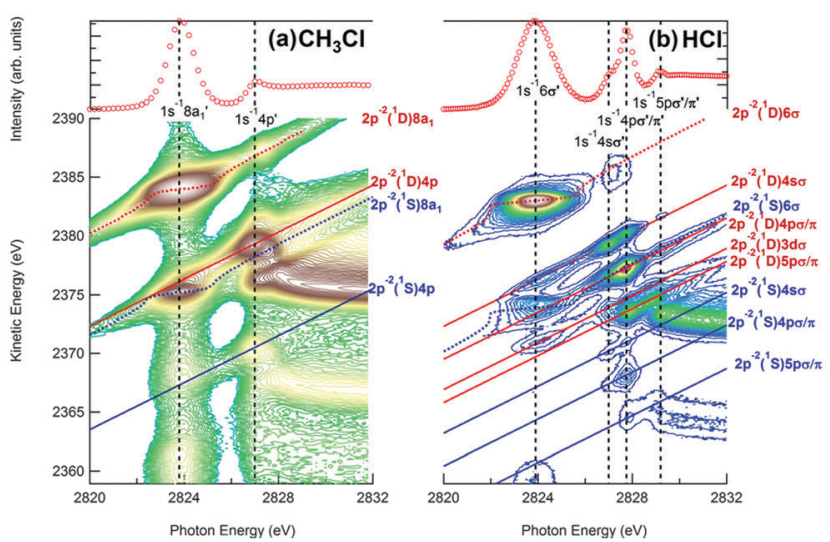

Fig. 2 2D-maps of Auger decay in $\mathrm{CH}_{3} \mathrm{Cl}$ (a) and $\mathrm{HCl}$ (b) after resonant excitation of the $\mathrm{Cl} \mathrm{K}$-shell. The kinetic energy of the Auger electron is plotted as a function of photon energy and the intensity is represented by a colorscale. The two corresponding PEY spectra are plotted above the 2D-maps as a more convenient way to show the intermediatestate resonances. The red lines are the $2 p^{-2}\left({ }^{1} D\right) \vee$ final states and the blue lines are the $2 p^{-2}\left({ }^{1} S\right) \vee$ final states. The dotted lines show the non-linear dispersion of the $2 \mathrm{p}^{-2}$ LUMO final states. A logarithmic colorscale was used in the case of $\mathrm{CH}_{3} \mathrm{Cl}$ to highlight the different spectroscopic terms. The solid lines show linearly-dispersive $2 \mathrm{p}^{-2}$ Rydberg final states.
In both molecules, the intermediate state $1 \mathrm{~s}^{-1}$ LUMO is dissociative. This is known from early RIXS measurements ${ }^{12,13}$ and is evidenced in Fig. 2 by the red and blue dotted lines representing the dispersion curves of the $2 \mathrm{p}^{-2}$ LUMO final state. The dispersion curves show the position of the Auger line as a function of photon energy. A characteristic 'S-shape', instead of a linear increase with the photon energy, is the evidence of nuclear dynamics: across the $1 \mathrm{~s}^{-1}$ LUMO resonance, the incident energy is not released entirely by the system in the form of Auger electron kinetic energy, but part of it is transmitted to the nuclei of the molecule, causing the elongation and finally the breaking of the chemical bond. ${ }^{1-10}$ Other features, represented by solid lines in Fig. 2, correspond to the $2 \mathrm{p}^{-2}$ Rydberg final states. They show a linear dispersion with photon energy: as the $1 \mathrm{~s}^{-1}$ Rydberg intermediate states are bound states, the nuclear wavepacket cannot propagate and all the energy available is carried by the Auger electron. Above the ionization potential (IP) - around a photon energy of $2829-2830 \mathrm{eV}$ - there are normal Auger lines, i.e. ionization of a 1s electron followed by KLL Auger decay. In this case, the Auger decay leads to a doubly charged ion in the final state and the lines are non-dispersive because the excess of incident energy goes into the kinetic energy of the photoelectron. Finally, around the threshold, one can observe post-collision interaction (PCI) effect, i.e. the three-body interaction between the slow photoelectron, the fast Auger electron and the ion. Here, PCI is observed as an exchange of energy between the Auger electron and the photoelectron, which distorts the energy distribution and shifts the Auger electron to higher energies close to threshold and may lead to a recapture of the photoelectron onto a Rydberg orbital, see for instance the $2 \mathrm{p}^{-2}\left({ }^{1} \mathrm{D}\right) 4 \mathrm{p} \sigma / \pi$ line for $\mathrm{HCl}$ in Fig. $2 \mathrm{~b}$. Note, that we use LS-coupling for the $2 \mathrm{p}^{-2}$ parent states since for third-row elements like $\mathrm{Cl}$ the spin-orbit interaction is known to be weak. ${ }^{31}$ The recapture effect has been recently observed under the same experimental conditions in Argon and has been studied in detail by Guillemin et al. ${ }^{32}$

From the PEY, we observe that the intensities of the $1 \mathrm{~s}^{-1} \mathrm{~V}^{\prime}$ resonances are comparable in $\mathrm{HCl}$, while in the case of $\mathrm{CH}_{3} \mathrm{Cl}$, the relative intensity of the $1 \mathrm{~s}^{-1}$ LUMO resonance is much higher than that of the $1 \mathrm{~s}^{-1}$ Rydberg intermediate states. In addition more discrete states are visible in $\mathrm{HCl}$, which partly explains the complexity of $\mathrm{HCl} 2 \mathrm{D}$-map compared to the $\mathrm{CH}_{3} \mathrm{Cl}$ one, see Fig. 2. In both $2 \mathrm{D}$-maps transitions to different $2 \mathrm{p}^{-2} \mathrm{~V}$ final states are observed. For $\mathrm{CH}_{3} \mathrm{Cl}$, the only visible final states (Fig. 2(a)) are $2 \mathrm{p}^{-2}\left({ }^{1} \mathrm{D}\right) 8 \mathrm{a}_{1}$ (red dotted line), $2 \mathrm{p}^{-2}\left({ }^{1} \mathrm{D}\right) 4 \mathrm{pa}_{1} / 4 \mathrm{pe}$ (red solid line), $2 \mathrm{p}^{-2}\left({ }^{1} \mathrm{~S}\right) 8 \mathrm{a}_{1}$ (blue dotted line) and $2 \mathrm{p}^{-2}\left({ }^{1} \mathrm{~S}\right) 4 \mathrm{pa}_{1} /$ 4 pe (blue solid line). In the 2D-map of $\mathrm{HCl}$ (from top to bottom in Fig. 2(b)), the red lines indicate the $2 \mathrm{p}^{-2}\left({ }^{1} \mathrm{D}\right) 6 \sigma, 2 \mathrm{p}^{-2}\left({ }^{1} \mathrm{D}\right) 4 \mathrm{~s} \sigma$, $2 \mathrm{p}^{-2}\left({ }^{1} \mathrm{D}\right) 4 \mathrm{p} \sigma / \pi, 2 \mathrm{p}^{-2}\left({ }^{1} \mathrm{D}\right) 3 \mathrm{~d} \sigma$ and $2 \mathrm{p}^{-2}\left({ }^{1} \mathrm{D}\right) 5 \mathrm{p} \sigma / \pi$ final states, whereas as the blue lines indicate the $2 \mathrm{p}^{-2}\left({ }^{1} \mathrm{~S}\right) 6 \sigma, 2 \mathrm{p}^{-2}\left({ }^{1} \mathrm{~S}\right) 4 \mathrm{~s} \sigma$, $2 \mathrm{p}^{-2}\left({ }^{1} \mathrm{~S}\right) 4 \mathrm{p} \sigma / \pi$ and $2 \mathrm{p}^{-2}\left({ }^{1} \mathrm{~S}\right) 5 \mathrm{p} \sigma / \pi$ final states. While the identification of almost all $2 \mathrm{p}^{-2}$ final states is obvious due to the resonance behavior manifested in the PEY spectrum, the assignment of the $2 \mathrm{p}^{-2}\left({ }^{1} \mathrm{D}\right) 3 \mathrm{~d} \sigma$ final state is more complex since it does not have a counterpart in the PEY. However, according to calculations, the $3 \mathrm{~d}$ states are expected between 
the $4 \mathrm{p}$ and the $5 \mathrm{p}$ states. ${ }^{33}$ Moreover, the assignment to the $\sigma$-like $3 \mathrm{~d}$ orbital can be derived from the resonant behavior in the $1 \mathrm{~s}^{-1} 6 \sigma^{\prime}$ energy region and the assumption of monopole shake transitions, i.e. shake transitions between molecular orbitals of the same symmetry $\lambda$.

Like in argon, ${ }^{22}$ we see local maxima on $2 \mathrm{p}^{-2}$ Rydberg states at the photon energy corresponding to $1 \mathrm{~s}^{-1} 6 \sigma^{\prime}$ resonance in HCl: at $h \nu=2823.8 \mathrm{eV}$, one can observe a local maximum of intensity at the kinetic energy $E_{\mathrm{k}} \approx 2375 \mathrm{eV}$, which corresponds to a transition to the $2 \mathrm{p}^{-2} 4 \mathrm{~s} \sigma$ final state. This is the evidence of a shake-up from the $1 \mathrm{~s}^{-1} 6 \sigma^{\prime}$ intermediate state to the $2 \mathrm{p}^{-2} 4 \mathrm{~s} \sigma$ final one. At the photon energy of $2823.8 \mathrm{eV}$ and the kinetic energy of $E_{\mathrm{k}} \approx 2372 \mathrm{eV}$, one can observe another local maximum that corresponds to the shake-up of an electron coming from the $6 \sigma^{\prime}$ orbital in the intermediate state, to the $4 \mathrm{p} \sigma$ orbital in the final state. To explore shakes and ELI effects, we have performed a series of simulations of the Auger spectra and of the pseudo-cross sections of different final states.

\section{Simulations}

The 2D-maps displayed in Fig. 2 can be described with the double differential cross section $\sigma\left(\omega, \omega^{\prime}\right)$ given by the KramersHeisenberg formula originally developed for RIXS. ${ }^{11,13,14}$ With $\omega$ being the excitation photon energy, $\omega^{\prime}$ being Auger electron energy the cross section can be written as

$$
\begin{aligned}
\sigma\left(\omega, \omega^{\prime}\right) & \propto\left|\sum_{\mathrm{c}} \frac{\langle\mathrm{f}|Q| \mathrm{c}\rangle\langle\mathrm{c}|D| \mathrm{o}\rangle}{\omega-\omega_{\mathrm{co}}+\frac{i \Gamma}{2}}\right|^{2} \delta\left(\omega-\omega^{\prime}-\omega_{\mathrm{fo}}\right) \\
& =\sigma(\omega) \delta\left(\omega-\omega^{\prime}-\omega_{\mathrm{fo}}\right) .
\end{aligned}
$$

In this equation $o$ and $f$ describe the initial state and the final state, respectively, while c stands for the different intermediate states. Moreover $D$ is the dipole operator for the excitation, $Q$ the Coulomb operator for the decay, $\omega_{\text {co }}$ the resonance energy for the core-excited state, $\omega_{\text {fo }}$ the transition frequency from the ground state to the final molecular state and $\Gamma$ the lifetime broadening of the core-excited state.

In this work, we have applied our model to fit the resonant Auger spectra measured at different photon energies as well as to obtain the pseudo-cross sections of the measured final states as a function of excitation energy. For both molecules resonant Auger spectra taken at different photon energies around the $1 \mathrm{~s}^{-1}$ LUMO resonances are shown in Fig. 3. In order to avoid too many fitting parameters, we restricted our fit to the $2 \mathrm{p}^{-2}\left({ }^{1} \mathrm{D}\right) \mathrm{V}$ series in $\mathrm{HCl}$ and $\mathrm{CH}_{3} \mathrm{Cl}$; as a result, minor contributions of the $2 \mathrm{p}^{-2}\left({ }^{1} \mathrm{~S}\right)$ LUMO final states of both molecules are not reproduced by the fit. In this figure the red dots represent our experimental points and the blue lines are the results of our simulation. The diagonal lines labeled (1) to (6) are displayed as a visual aid to identify the resonances in both molecules: $2 \mathrm{p}^{-2}\left({ }^{1} \mathrm{D}\right) 4 \mathrm{pe} / \mathrm{a}_{1}, \quad 2 \mathrm{p}^{-2}\left({ }^{1} \mathrm{D}\right) 8 \mathrm{a}_{1}$ for $\mathrm{CH}_{3} \mathrm{Cl}$ and $2 \mathrm{p}^{-2}\left({ }^{1} \mathrm{D}\right) 3 \mathrm{~d} \sigma$, $2 \mathrm{p}^{-2}\left({ }^{1} \mathrm{D}\right) 4 \mathrm{p} \sigma / \pi, 2 \mathrm{p}^{-2}\left({ }^{1} \mathrm{D}\right) 4 \mathrm{~s} \sigma$ and $2 \mathrm{p}^{-2}\left({ }^{1} \mathrm{D}\right) 6 \sigma$ for $\mathrm{HCl}$.
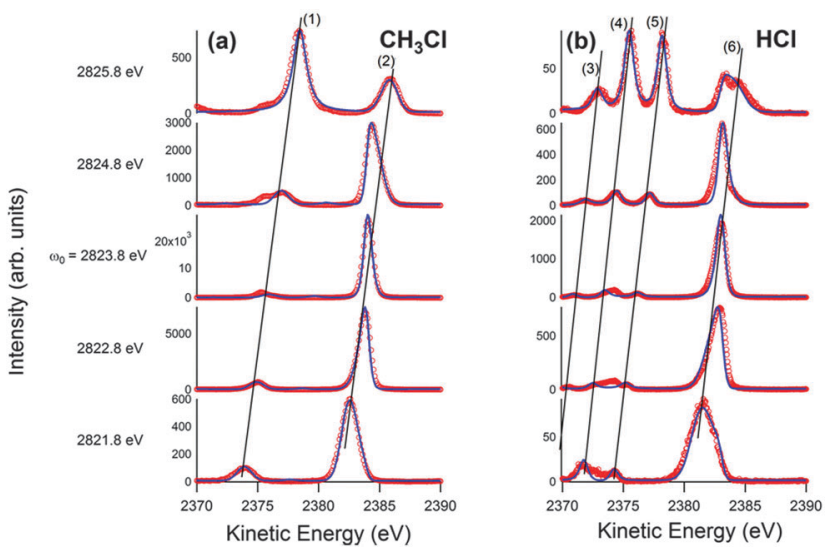

Fig. 3 Auger spectra of $\mathrm{CH}_{3} \mathrm{Cl}(\mathrm{a})$ and $\mathrm{HCl}$ (b) for several photon energies around the $1 \mathrm{~s}^{-1}$ LUMO resonance. The dots represent the experimental points and the solid lines result from our simulation. The peak (1) is $2 p^{-2}\left({ }^{1} D\right) 4 p$ and the peak (2) is the $2 p^{-2}\left({ }^{1} D\right) 8 a_{1}$ of $\mathrm{CH}_{3} \mathrm{Cl}$. The peaks (3) to (6) are the final states $2 p^{-2}\left({ }^{1} D\right) 3 d \sigma, 2 p^{-2}\left({ }^{1} D\right) 4 p \sigma / \pi, 2 p^{-2}\left({ }^{1} D\right) 4 s \sigma$ and $2 \mathrm{p}^{-2}\left({ }^{1} \mathrm{D}\right) 6 \sigma$, respectively, of $\mathrm{HCl}$.

The pseudo-cross sections for one particular final state $n \lambda$ as a function of photon energy, $\sigma_{n \lambda}^{+}(\omega)$, are displayed in Fig. 4 (for $\mathrm{HCl}$ ) and Fig. 5 (for $\mathrm{CH}_{3} \mathrm{Cl}$ ). In the present case these final states are of the type $2 \mathrm{p}^{-2}\left({ }^{1} \mathrm{~S},{ }^{1} \mathrm{D}\right) n \lambda$ with $n=6,4$ and $\lambda$ (describes the symmetry of the orbitals) $=\sigma, \mathrm{s} \sigma, \mathrm{p} \sigma, \mathrm{p} \pi$ for $\mathrm{HCl}$ and $n=8,4$ and $\lambda=\mathrm{a}_{1}$, pe and $\mathrm{pa}_{1}$ for $\mathrm{CH}_{3} \mathrm{Cl}$. A particular final state $n \lambda$ can be found along the diagonal lines indicated in Fig. 2. To derive the pseudo-cross sections $\sigma_{n \lambda}^{+}(\omega)$ from the 2D-maps one has to integrate over a suitable range of Auger energies $\omega^{\prime}$ around the diagonal lines.

In the following, we first describe the general points directly related to eqn (2). Then, we discuss in detail the simulations of the resonant Auger spectra and the pseudo-cross sections $\sigma_{n \lambda}^{+}$ $(\omega)$. The above described states o, c, and f depend on both the electronic and the nuclear coordinates. In the Born-Oppenheimer approximation each of these states can be described as a product of an electronic and a vibrational wavefunction so that $\sigma(\omega)$ can be written as

$$
\sigma(\omega)=\sigma_{\mathrm{DIR}}(\omega)+\sigma_{\mathrm{ELI}}(\omega)+\sigma_{\mathrm{VLI}}(\omega)+\sigma_{\mathrm{ELI}, \mathrm{VLI}}(\omega) .
$$

Here $\sigma_{\text {DIR }}(\omega)$ describes the direct term which corresponds to a decay without interferences. The contributions $\sigma_{\mathrm{ELI}}(\omega), \sigma_{\mathrm{VLI}}(\omega)$, and $\sigma_{\text {ELI,VLI }}(\omega)$ originate from the cross terms and describe ELI, vibrational lifetime interferences (VLI), and a mixture of electronic and vibrational lifetime interference, respectively. For details of the expansion of $\sigma(\omega)$ and in particular for VLI, see Püttner $e t a l .^{34}$ and Poygin et $\mathrm{al}^{35}$ In the present analysis, only the direct terms and the ELI terms are taken into account since the individual vibrational states cannot be resolved.

The Kramers-Heisenberg formula given in eqn (2) is exact when the direct terms in the spectrum can be described with Lorentzian lineshapes which are derived from the denominators in this equation. This is given for the case that each intermediate and final state is an isolated level with respect to the electronic and vibrational parts. However, the presence of a continuum of 


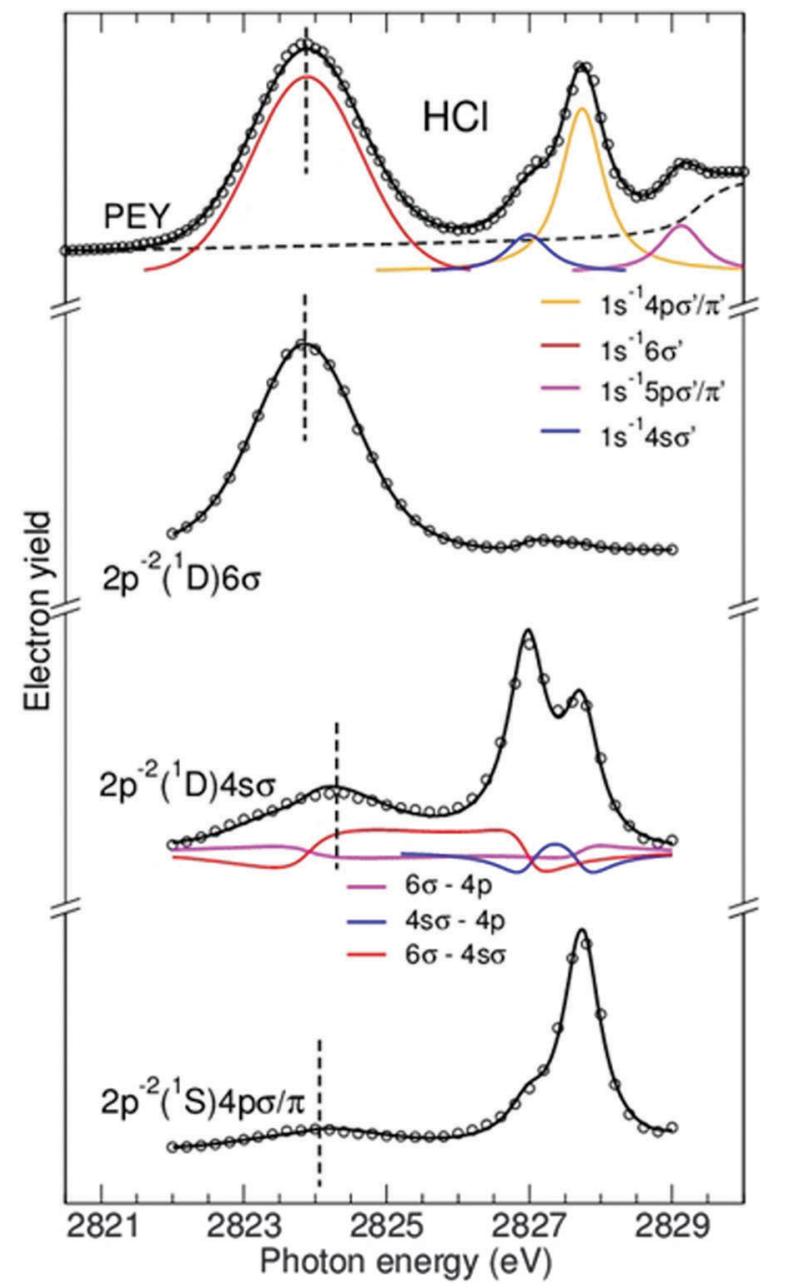

Fig. 4 The PEY spectrum as well as the pseudo-cross sections leading to the final states $2 p^{-2} 6 \sigma, 2 p^{-2} 4 s \sigma$, and $2 p^{-2} 4 p$ in $\mathrm{HCl}$. The solid lines through the data points represent the fit results. The subspectra below the PEY spectrum indicate the contributions of the individual $\mathrm{Cl}_{1} \mathrm{~s}^{-1}$ core excited states and the subspectra below the cross section of the $2 \mathrm{p}^{-2} 4 \mathrm{~s} \sigma$ final state show the ELI contributions.

vibrational levels can result in distortion of the Lorentzian shape, which is further affected by the ELI contributions. To account for these effects, we applied a simplified approach illustrated below for the case of two discrete intermediate states. In this case the cross section of eqn (2) can be written as

$$
\begin{aligned}
\sigma(\omega)= & \left|\frac{K_{1}}{\omega-\tau_{1}+i \Gamma_{1} / 2}+\frac{K_{2}}{\omega-\tau_{2}+i \Gamma_{2} / 2}\right|^{2} \\
= & \operatorname{Lor}\left(\Gamma_{1}, \tau_{1}, \omega\right)+\operatorname{Lor}\left(\Gamma_{2}, \tau_{2}, \omega\right) \\
& +\frac{\left(K_{1} K_{2}{ }^{*}+K_{1}{ }^{*} K_{2}\right) \cdot\left(\left(\omega-\tau_{1}\right)\left(\omega-\tau_{2}\right)+\frac{\Gamma_{1} \cdot \Gamma_{2}}{4}\right)}{\left[\left(\omega-\tau_{1}\right)^{2}+\frac{\Gamma_{1}{ }^{2}}{4}\right]\left[\left(\omega-\tau_{2}\right)^{2}+\frac{\Gamma_{2}^{2}}{4}\right]} .
\end{aligned}
$$

Here, $\tau_{i}$ and $\Gamma_{i}$ are the resonance energy and the natural broadening of the $i$ th resonance while $K_{i}=\left\langle\mathrm{o}|D| \mathrm{c}_{i}\right\rangle\left\langle\mathrm{c}_{i}|Q| \mathrm{f}\right\rangle$ is

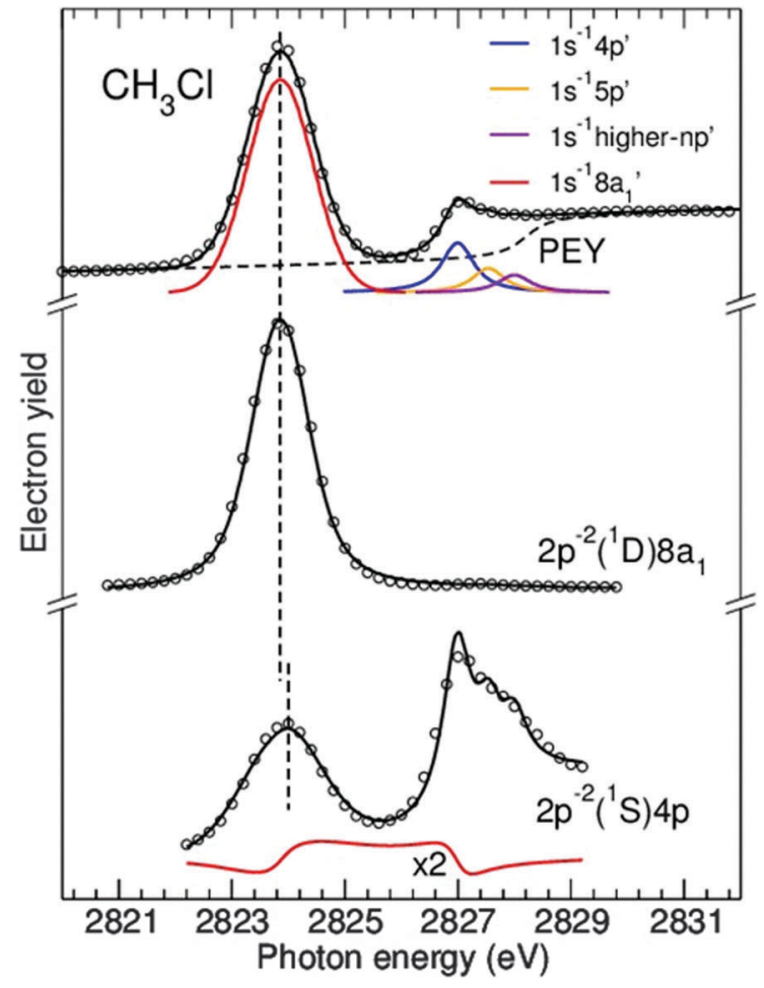

Fig. 5 The PEY spectrum as well as the pseudo-cross sections leading to the final states $2 p^{-2} 8 a_{1}$ and $2 p^{-2} 4 p$ in $\mathrm{CH}_{3} \mathrm{Cl}$. The solid lines through the data points represent the fit results. The subspectra below the PEY spectrum indicate the contributions of the individual $\mathrm{Cl}_{1} \mathrm{~s}^{-1}$ core excited states and the subspectra below the cross section of the $2 \mathrm{p}^{-2} 4 \mathrm{p}$ final state show the ELI contributions.

the product of the matrix elements for the excitation and the decay. Moreover,

$$
\operatorname{Lor}\left(\Gamma_{i}, \tau_{i}, \omega\right)=\left|\frac{K_{i}}{\omega-\tau_{i}+i \Gamma_{i} / 2}\right|^{2}=\frac{\left|K_{i}\right|^{2}}{\left(\omega-\tau_{i}\right)^{2}+\Gamma_{i}^{2} / 4}
$$

describes the lineshape of the $i$ th resonance. Note that in $K_{1} K_{2}^{*}=\left\langle\mathrm{o}|D| \mathrm{c}_{1}\right\rangle\left\langle\mathrm{c}_{1}|Q| \mathrm{f}\right\rangle\left\langle\mathrm{f}|Q| \mathrm{c}_{2}\right\rangle\left\langle\mathrm{c}_{2}|D| \mathrm{o}\right\rangle$ each wavefunction contributes in the form of a bra- and a ket-vector so that possible phases cancel out. Because of this the wavefunctions can be chosen without the loss of generality such that the $K_{i}$ become real quantities. In this way we can write

$$
K_{i}= \pm \sqrt{\operatorname{Lor}\left(\Gamma_{i}, \tau_{i}, \omega\right)\left(\left(\omega-\tau_{i}\right)^{2}+\Gamma_{i}^{2} / 4\right)}
$$

and

$$
f\left(\tau_{1}, \tau_{2}, \Gamma_{1}, \Gamma_{2}, \omega\right)=\frac{\left(\omega-\tau_{1}\right)\left(\omega-\tau_{2}\right)+\frac{\Gamma_{1} \cdot \Gamma_{2}}{4}}{\sqrt{\left[\left(\omega-\tau_{1}\right)^{2}+\frac{\Gamma_{1}^{2}}{4}\right]\left[\left(\omega-\tau_{2}\right)^{2}+\frac{\Gamma_{2}^{2}}{4}\right]}},
$$

which leads to

$$
\begin{aligned}
\sigma(\omega)= & \operatorname{Lor}\left(\Gamma_{1}, \tau_{1}, \omega\right)+\operatorname{Lor}\left(\Gamma_{2}, \tau_{2}, \omega\right) \\
& \pm 2 \sqrt{\operatorname{Lor}\left(\Gamma_{1}, \tau_{1}, \omega\right) \cdot \operatorname{Lor}\left(\Gamma_{2}, \tau_{2}, \omega\right)} \cdot f\left(\tau_{1}, \tau_{2}, \Gamma_{1}, \Gamma_{2}, \omega\right)
\end{aligned}
$$


The two different signs in the last line of eqn (8) are due to the fact that the present empirical approach provides only the absolute value of the matrix elements but not the matrix elements themselves. We would like to point out that a similar situation occurs also in the case of VLI where the interference term can also be calculated directly by using one-dimensional vibrational wavefunctions. ${ }^{36}$ This allows an interpretation of the sign and provides additional information about the process of excitation and decay; for details see Püttner et $a .^{34,36}$ For additional information concerning the origin of the sign in the case of ELI see the discussion below eqn (11).

For ELI, it is not sufficient to consider the ionic final state. Instead, the total electronic final state including the ionic final state and the outgoing Auger electron has to be taken into account. Since the Auger electron wavefunction can have different symmetries, one ionic final state can lead to different total electronic final states. As has been shown in Püttner et al. ${ }^{34}$ and Poygin et al., ${ }^{35}$ the ELI-term is reduced compared to the value 2 . $\sqrt{\operatorname{Lor}\left(\Gamma_{1}, \tau_{1}, \omega\right) \cdot \operatorname{Lor}\left(\Gamma_{2}, \tau_{2}, \omega\right)} \cdot f\left(\tau_{1}, \tau_{2}, \Gamma_{1}, \Gamma_{2}, \omega\right)$ obtained from the present fit approach if different total final states are preferentially populated by different electronic intermediate states.

When different intermediate and/or final states overlap (like for instance in the present case the $\mathrm{Cl} 1 \mathrm{~s}^{-1} 4 \mathrm{p} \sigma^{\prime} / \pi^{\prime}$ excitation in the PEY or the $2 \mathrm{p}^{-2} 4 \mathrm{p} \sigma / \pi$ pseudo-cross section presented in Fig. 4) the individual ELI-terms cannot be resolved. As will be discussed in detail below, this leads also to a reduction of the ELI-terms obtained in the present fit approach. To account for this reduction of the ELI-terms the additional fit parameter $\beta_{1,2}$ with $-1 \leq \beta_{1,2} \leq 1$ has to be taken into account so that eqn (8) results in

$$
\begin{aligned}
\sigma(\omega)= & \operatorname{Lor}\left(\Gamma_{1}, \tau_{1}, \omega\right)+\operatorname{Lor}\left(\Gamma_{2}, \tau_{2}, \omega\right) \\
& +2 \cdot \beta_{1,2} \cdot \sqrt{\operatorname{Lor}\left(\Gamma_{1}, \tau_{1}, \omega\right) \operatorname{Lor}\left(\Gamma_{2}, \tau_{2}, \omega\right)} \\
& \times f\left(\tau_{1}, \tau_{2}, \Gamma_{1}, \Gamma_{2}, \omega\right) .
\end{aligned}
$$

With $\beta_{1,2}= \pm 1$ the ELI term reaches its maximum and eqn (9) reduces to eqn (8). The value $\beta_{1,2}=0$ indicates the absence of interference as it occurs, for example, in the case of well-separated intermediate states with different symmetries $\lambda$, while all the other values of $\beta_{1,2}$ indicate a reduction of the ELI term caused by overlapping states with different symmetries. We will show below how to utilize the value of $\beta_{1,2}$ to determine the intensity ratio of these unresolved overlapping states.

Up to now we have considered transitions between isolated levels so that the lineshapes can be described with Lorentzian functions. In the case of a dissociative intermediate and a dissociative final state with almost parallel potential energy curves the influence of the vibrational wavefunction including the nuclear dynamics on the double differential cross section $\sigma\left(\omega, \omega^{\prime}\right)$ given in eqn (2) can be approximated with the formula ${ }^{11}$

$$
\sigma\left(\omega, \omega^{\prime}\right)=\sigma\left(\Omega+\omega_{\mathrm{co}}, \Omega^{\prime}+\omega_{\mathrm{cf}}\right) \propto \frac{\exp \left(-\left[\frac{\Omega^{\prime}-\Omega}{\Delta}\right]^{2} \ln 2\right)}{\Omega^{\prime 2}+\frac{\Gamma^{2}}{4}} .
$$

In the present study this simplified formula has been applied to the resonant Auger decay of the dissociative intermediate state $1 \mathrm{~s}^{-1}$ LUMO to the dissociative final state $2 \mathrm{p}^{-2}$ LUMO. Here $\Omega=\omega-\omega_{\text {co }}$ is the detuning of the incident photon energy from the resonance energy of the core-excited state $\omega_{\text {co }}$ and $\Omega^{\prime}=\omega^{\prime}-\omega_{\text {cf }}$ the detuning of the kinetic energy of the Auger electron from the resonance emission energy $\omega_{\text {cf }}$. Moreover, $\Delta$ is the half width at half maximum of the Franck-Condon (FC) distribution between the ground and excited states. In our simulations we have used values found in the literature, namely $\Delta=0.65 \mathrm{eV}^{13}$ in the case of $\mathrm{CH}_{3} \mathrm{Cl}$ and $\Delta=0.84 \mathrm{eV}^{37}$ in the case of $\mathrm{HCl}$. The value $\Gamma=0.65 \mathrm{eV}$ has been extracted from the analytical fit using eqn (10) and is in agreement with Campbell and $\operatorname{Papp}^{38}(0.574 \mathrm{eV} \pm 0.14 \mathrm{eV})$. In eqn (10), the double differential cross section is reduced to the product of a Gaussian which describes the nuclear dynamics and a Lorentzian related to the lifetime broadening of the core-excited state.

In the following, we shall specify the procedures for simulating resonant Auger spectra partially presented in Fig. 3 and the pseudo-cross sections presented in Fig. 4 and 5. The resonant Auger spectra are fitted with eqn (2) and in the case of the $\mathrm{Cl}$ $1 \mathrm{~s}^{-1}$ LUMO $\rightarrow \mathrm{Cl} 2 \mathrm{p}^{-2}$ LUMO transitions with eqn (10).

To study the role of ELI in the pseudo-cross sections $\sigma_{n \lambda}^{+}(\omega)$ in more detail, the intensities of the different $2 \mathrm{p}^{-2}\left({ }^{1} \mathrm{~S}\right) n \lambda$ and $2 \mathrm{p}^{-2}\left({ }^{1} \mathrm{D}\right) n \lambda$ final states were extracted from the Auger spectra by less restrictive fits using Voigt functions for each final state. To analyze these pseudo-cross sections we applied eqn (4) taking into account all possible intermediate states:

$$
\begin{aligned}
\sigma_{n \lambda}^{+}(\omega) & =\left|\sum_{m} \frac{\left\langle\text { g.s. }|D| 1 \mathrm{~s}^{-1} m \lambda^{\prime}\right\rangle\left\langle 1 \mathrm{~s}^{-1} m \lambda^{\prime}|Q| 2 \mathrm{p}^{-2} n \lambda\right\rangle}{\omega-\tau_{m \lambda^{\prime}}+i \Gamma_{m \lambda^{\prime}} / 2}\right|^{2} \\
& =\Gamma_{\mathrm{KLL}}\left|\sum_{m} \frac{\left\langle\text { g.s. }|D| 1 \mathrm{~s}^{-1} m \lambda^{\prime}\right\rangle\left\langle m \lambda^{\prime} \mid n \lambda\right\rangle}{\omega-\tau_{m \lambda^{\prime}}+i \Gamma_{m \lambda^{\prime}} / 2}\right|^{2} .
\end{aligned}
$$

Here $m$ describes the orbitals of the excited electron in the presence of a 1s core-hole and $n$ refers to the orbitals of the excited electron in the $2 \mathrm{p}^{-2}$ double core-hole final state. Following Armen et al. ${ }^{23}$ and Kukk et al., ${ }^{39}$ the spectator KLL Auger decay can be considered independent of the orbital of the spectator electron so that $\left\langle 1 \mathrm{~s}^{-1} m \lambda^{\prime}|Q| 2 \mathrm{p}^{-2} n \lambda\right\rangle=$ $\left\langle 1 \mathrm{~s}^{-1}|Q| 2 \mathrm{p}^{-2}\right\rangle\left\langle m \lambda^{\prime} \mid n \lambda\right\rangle$, resulting in $\Gamma_{\mathrm{KLL}}=\left|\left\langle 1 \mathrm{~s}^{-1}|Q| 2 \mathrm{p}^{-2}\right\rangle\right|^{2}$ being the partial rate for the $\mathrm{K}-\mathrm{L}_{2,3} \mathrm{~L}_{2,3}$ Auger decay. Because of the difference in the excited and final core-hole states, the orbital wavefunctions $\left|m \lambda^{\prime}\right\rangle$ are not orthogonal to $|n \lambda\rangle$ so that the overlap matrix elements $\left\langle m \lambda^{\prime} \mid n \lambda\right\rangle$ describe the possible shake processes during the Auger decay. Note that we assume the sudden approximation so that only monopole shake transitions occur; this allows neglecting the summation over all possible symmetries $\lambda$ of the excited electron in eqn (11).

An expansion of the second line of eqn (11) shows that the sign of the ELI-term depends only on the signs of the dipole matrix elements $\left\langle\right.$ g.s. $\left.|D| 1 \mathrm{~s}^{-1} m \lambda^{\prime}\right\rangle$ and the overlap matrix elements $\left\langle m \lambda^{\prime} \mid n \lambda\right\rangle$, but not on the sign of the Coulomb matrix elements $\left\langle 1 \mathrm{~s}^{-1}|Q| 2 \mathrm{p}^{-2}\right\rangle$. This has been investigated theoretically in more detail for krypton by Kukk et al. ${ }^{39}$ In detail, Kukk et al. calculated 
the dipole matrix elements for the $\left\langle\right.$ g.s. $\left.|D| 3 \mathrm{~d}^{-1} 5 \mathrm{p}^{\prime}, 6 \mathrm{p}^{\prime}\right\rangle$ excitations as well as the $\left\langle m p^{\prime} \mid n p\right\rangle$ overlap integrals for $m=5,6$ and $n=5,6,7$. For krypton it was found that both dipole matrix elements had a positive sign. The same holds for the overlap integrals in the case of a shake-down or spectator Auger decay, while their sign is negative for shake-up Auger decays. These findings allowed reproducing the signs of the ELI-contributions in the experimental $4 \mathrm{p}^{-2} n \mathrm{p}$ partial cross sections. We expect that in the present case of molecules the signs of $\beta_{1,2}$ provide similar information. However, similar theoretical investigations of the electronic wavefunctions are beyond the scope of the present work.

For fitting the LUMO contributions in the pseudo-cross sections $\sigma_{n \lambda}^{+}(\omega)$ the double differential cross section described by eqn (10) was integrated over a suitable range of kinetic energies around the red dotted lines given in Fig. 2, i.e.

$$
\begin{aligned}
\sigma_{\mathrm{LUMO}}(\omega) & =\int \sigma\left(\omega, \omega^{\prime}\right) \mathrm{d} \omega^{\prime} \propto \int \frac{\exp \left(-\left[\frac{\Omega^{\prime}-\Omega}{\Delta}\right]^{2} \ln 2\right)}{\Omega^{\prime 2}+\frac{\Gamma^{2}}{4}} \mathrm{~d} \Omega^{\prime} . \\
& =\operatorname{LS}(\Gamma, \tau, \omega)
\end{aligned}
$$

Here $\operatorname{LS}(\Gamma, \tau, \omega)$ describes a lineshape different from a Lorentzian lineshape so that eqn (4) can be approximated by

$$
\begin{aligned}
\sigma(\omega)= & \operatorname{LS}\left(\Gamma_{1}, \tau_{1}, \omega\right)+\operatorname{Lor}\left(\Gamma_{2}, \tau_{2}, \omega\right) \\
& +2 \cdot \beta_{1,2} \cdot \sqrt{\operatorname{LS}\left(\Gamma_{1}, \tau_{1}, \omega\right) \cdot \operatorname{Lor}\left(\Gamma_{2}, \tau_{2}, \omega\right)} \\
& \times f\left(\tau_{1}, \tau_{2}, \Gamma_{1}, \Gamma_{2}, \omega\right) .
\end{aligned}
$$

Using eqn (13) we can describe (in the pseudo-cross section) the ELI contributions between a discrete Rydberg state and a continuum of vibrational levels in the LUMO in an approximate way. In principle $\operatorname{LS}(\Gamma, \tau, \omega)$ represents a continuum of Lorentzian functions affecting also the term $f\left(\tau_{1}, \tau_{2}, \Gamma_{1}, \Gamma_{2}, \omega\right)$. However, simulations using either $\operatorname{LS}(\Gamma, \tau, \omega)$ or a finite number of Lorentzian lines provide semiquantitative agreement, thus confirming our approximation. In addition to the described approach for the pseudo-cross section, an arctangent function was included to represent the unresolved Rydberg states as well as the continuum.

Finally, all fit results shown in Fig. 3-5 were convoluted using a Gaussian function of $460 \mathrm{meV}$ FWHM for $\mathrm{HCl}$ and $440 \mathrm{meV}$ for $\mathrm{CH}_{3} \mathrm{Cl}$ to account for the photon bandwidth as well as the analyzer resolution.

\section{Results and discussion}

Fig. 4 shows the PEY spectrum in $\mathrm{HCl}$ as well as the pseudocross sections leading to the final states $2 \mathrm{p}^{-2} 6 \sigma, 2 \mathrm{p}^{-2} 4 \mathrm{~s} \sigma$, and $2 \mathrm{p}^{-2} 4 \mathrm{p}$. The four spectra were fitted in parallel and the fit result is represented by the black solid lines through the data points. For the PEY spectrum the contributions of the individual coreexcited intermediate states below the $\mathrm{Cl} 1 \mathrm{~s}^{-1}$ ionization threshold are indicated by the subspectra.
Table 1 Summary of the fit results obtained for $\beta_{i, j}$ in $\mathrm{HCl}$

\begin{tabular}{lccc}
\hline Final state & $\beta_{6 \sigma, 4 \mathrm{~s} \sigma}$ & $\beta_{6 \sigma, 4 \mathrm{p} \sigma / \pi}$ & $\beta_{4 \mathrm{~s} \sigma, 4 \mathrm{p} \sigma / \pi}$ \\
\hline $2 \mathrm{p}^{-2} 6 \sigma$ & 0.57 & -0.03 & -0.45 \\
$2 \mathrm{p}^{-2} 4 \mathrm{~s} \sigma$ & -1.00 & 0.47 & -0.27 \\
$2 \mathrm{p}^{-2} 4 \mathrm{p} \sigma / \pi$ & -1.00 & 0.14 & -0.18
\end{tabular}

For the pseudo-cross section spectra the $6 \sigma-4 \mathrm{~s} \sigma, 6 \sigma-4 \mathrm{p}$, and $4 \mathrm{~s} \sigma-4 \mathrm{p} \sigma / \pi \mathrm{ELI}$ terms are taken into account. In the case of the $2 \mathrm{p}^{-2} 4 \mathrm{~s} \sigma$ pseudo-cross section these contributions are indicated by the subspectra in color and the corresponding $\beta_{i, j}$ values are summarized in Table 1 . As indicated by the dashed vertical lines, ELI leads to different peak positions for the $6 \sigma$ state in the various pseudo-cross sections. Similar shifts in the peak position are also present for the $4 \mathrm{~s} \sigma$ and the $4 \mathrm{p}$ states. They are, however, too small to be observed directly due to the overlapping contributions of the $4 \mathrm{~s} \sigma-4 \mathrm{p}$ ELI term.

In the following we shall discuss the values for $\beta_{i, j}$ obtained from the fit analysis. When the factors $\beta_{i, j}= \pm 1$, the ELI term is maximal. This can only occur when two or more intermediate states decay exclusively into the same final state, whereas in the case of overlapping final states $2 \mathrm{p}^{-2} 4 \mathrm{p} \sigma / \pi, \beta_{i, j}$ can take any value in the interval $[-1 ; 1]$. Here we will discuss only the aspects of the ELI relevant for the present case. For a more general discussion see Püttner et al. ${ }^{34}$ and Poygin et al. ${ }^{35}$

Let us discuss the obtained values of the factors $\beta_{6 \sigma, 4 \mathrm{~s} \sigma}$ in the pseudo-cross sections $2 \mathrm{p}^{-2} 4 \mathrm{~s} \sigma$ and $2 \mathrm{p}^{-2} 6 \sigma$. In both cases, $\beta_{6 \sigma, 4 \mathrm{~s} \sigma}$ is close to \pm 1 which can be interpreted as follows. The ELI between the states $6 \sigma$ and $4 \mathrm{~s} \sigma$ in the $2 \mathrm{p}^{-2} 4 \mathrm{~s} \sigma$ partial cross section, i.e. the population of the $2 \mathrm{p}^{-2} 4 \mathrm{~s} \sigma$ final state via the $1 \mathrm{~s}^{-1} 6 \sigma^{\prime}$ and $1 \mathrm{~s}^{-1} 4 \mathrm{~s} \sigma^{\prime}$ intermediate states, can be described using the matrix elements

$$
\begin{aligned}
\langle\text { g.s. }| & D\left|1 \mathrm{~s}^{-1} 6 \sigma^{\prime}\right\rangle\left\langle 1 \mathrm{~s}^{-1} 6 \sigma^{\prime}|Q| 2 \mathrm{p}^{-2} 4 \mathrm{~s} \sigma\right\rangle \\
\quad \times & \left\langle 2 \mathrm{p}^{-2} 4 \mathrm{~s} \sigma|Q| 1 \mathrm{~s}^{-1} 4 \mathrm{~s} \sigma^{\prime}\right\rangle\left\langle 1 \mathrm{~s}^{-1} 4 \mathrm{~s} \sigma^{\prime}|D| \text { g.s. }\right\rangle
\end{aligned}
$$

Here g.s. stands for the ground state. Moreover,

$$
\left\langle\text { g.s. }|D| 1 \mathrm{~s}^{-1} 6 \sigma^{\prime}\right\rangle\left\langle 1 \mathrm{~s}^{-1} 6 \sigma^{\prime}|Q| 2 \mathrm{p}^{-2} 4 \mathrm{~s} \sigma\right\rangle=\sqrt{I\left(6 \sigma^{\prime}, 4 \mathrm{~s} \sigma\right)}
$$

and

$$
\left\langle 2 \mathrm{p}^{-2} 4 \mathrm{~s} \sigma|Q| 1 \mathrm{~s}^{-1} 4 \mathrm{~s} \sigma^{\prime}\right\rangle\left\langle 1 \mathrm{~s}^{-1} 4 \mathrm{~s} \sigma^{\prime}|D| \text { g.s. }\right\rangle=\sqrt{I\left(4 \mathrm{~s} \sigma^{\prime}, 4 \mathrm{~s} \sigma\right)}
$$

are the amplitudes of the direct decay without interference, described by a product of a Gaussian and a Lorentzian for the $6 \sigma$ (eqn (10)) and by a Lorentzian for the $4 \mathrm{~s} \sigma$ state.

In the fit analysis the ELI term is described by

$$
\sqrt{I\left(6 \sigma^{\prime}, 4 \mathrm{~s} \sigma\right)} \sqrt{I\left(4 \mathrm{~s} \sigma^{\prime}, 4 \mathrm{~s} \sigma\right)} \beta_{6 \sigma, 4 \mathrm{~s} \sigma} .
$$

In the present case the final state is populated by two different intermediate states that decay to the given final state. Because of this, no reduction of the ELI contributions is expected resulting in $\beta_{6 \sigma, 4 s \sigma}= \pm 1$, in agreement with the fit result. Note that the sign + or - results from the signs of the individual dipole and overlap matrix elements in the ELI terms, see above. These signs can be obtained from calculations that are beyond the scope of the present studies. 
In principle, for the final state of the Auger decay and for the analysis of ELI, one has to take into account the outgoing Auger electron. For instance, the decay of the $\mathrm{Cl} 1 \mathrm{~s}^{-1} 4 \mathrm{~s} \sigma^{\prime}\left({ }^{1} \Sigma\right)$ intermediate state to the $2 \mathrm{p}^{-2}\left({ }^{1} \mathrm{D}\right) 4 \mathrm{~s} \sigma$ ionic final state leads to different total final states including the Auger electron. Taking into account only monopole Auger transitions one obtains the total final states $2 \mathrm{p}^{-2}\left({ }^{1} \Delta\right) 4 \mathrm{~s} \sigma \varepsilon \delta\left({ }^{1} \Sigma\right), 2 \mathrm{p}^{-2}\left({ }^{1} \Pi\right) 4 \mathrm{~s} \sigma \varepsilon \pi\left({ }^{1} \Sigma\right)$, and $2 \mathrm{p}^{-2}\left({ }^{1} \Sigma\right) 4 \mathrm{~s} \sigma \varepsilon \sigma\left({ }^{1} \Sigma\right)$; here $\varepsilon \lambda$ describes the outgoing Auger electron. These three final states overlap in the 2D-map presented in Fig. $2 \mathrm{~b}$ so that the $2 \mathrm{p}^{-2} 4 \mathrm{~s} \sigma$ pseudo-cross section actually consists of three overlapping pseudo-cross sections. However, this overlap has no influence on the ELI term if the decay branching ratio of the $1 \mathrm{~s}^{-1} 6 \sigma^{\prime}$ and $1 \mathrm{~s}^{-1} 4 \mathrm{~s} \sigma^{\prime}$ intermediate states to the total final state is independent of the excited $6 \sigma^{\prime}$ and $4 s \sigma^{\prime}$ electron in the intermediate state. In this case the branching ratio from a given intermediate state to a given final state can be described as proportional to the corresponding intensity observable in the pseudo-cross section. This results in an additional proportionality factor $\alpha_{i}^{2}$ in the ELI terms so that a summation over all final states results in $\sum_{i} \alpha_{i}^{2}=1$.

Based on the assumption of monopole shake transitions during the Auger decay the $2 \mathrm{p}^{-2} 6 \sigma$ and $2 \mathrm{p}^{-2} 4 \mathrm{~s} \sigma$ pseudo-cross sections can only be populated via 1 s excitations into orbitals with $\sigma$ symmetry. These are in the studied energy region only $1 \mathrm{~s}^{-1} 6 \sigma^{\prime}, 1 \mathrm{~s}^{-1} 4 \mathrm{~s} \sigma^{\prime}$, and $1 \mathrm{~s}^{-1} 4 \mathrm{p} \sigma^{\prime}$ so that the above given arguments result in \pm 1 for the $\beta_{i, j}$ - factors present in the discussed pseudocross sections. Indeed $\beta_{6 \sigma, 4 \mathrm{~s} \sigma}$ for the $2 \mathrm{p}^{-2} 6 \sigma$ and $2 \mathrm{p}^{-2} 4 \mathrm{~s} \sigma$ pseudocross sections are close to \pm 1 as shown in Table 1 . Note that the pseudo-cross sections could be extracted from the 2D-maps only up to a photon energy of $2828.25 \mathrm{eV}$ so that the ELI terms including the higher Rydberg states and the continuum states could not be taken into account. This influences in particular the region of the $1 \mathrm{~s}^{-1} 4 \mathrm{~s} \sigma^{\prime}$ and $1 \mathrm{~s}^{-1} 4 \mathrm{p} \sigma^{\prime} / \pi^{\prime}$ intermediate states due to their vicinity to the neglected states.

In the case of the $2 \mathrm{p}^{-2} 4 \mathrm{p} \sigma / \pi$ pseudo-cross section the situation is more complicated since two different final states overlap and cannot be resolved. For the $6 \sigma-4 \mathrm{~s} \sigma$ ELI term the situation is still rather simple since monopole Auger transitions lead only to the $2 \mathrm{p}^{-2} 4 \mathrm{p} \sigma$ final state so that the above given arguments still hold and $\beta_{6 \sigma, 4 \mathrm{~s} \sigma}= \pm 1$ can be expected, in agreement with the fit result. For the ELI terms including the $1 \mathrm{~s}^{-1} 4 \mathrm{p} \sigma^{\prime} / \pi^{\prime}$ intermediate states, the situation is much more complex. In the case of monopole transitions, only ELI between $4 \mathrm{p} \sigma$ and $6 \sigma$ or $4 \mathrm{~s} \sigma$ can occur, but not between $4 \mathrm{p} \pi$ and $6 \sigma$ or $4 \mathrm{~s} \sigma$. As a result, the ELI term between $6 \sigma$ and $4 \mathrm{p} \sigma$ has to be described by

$$
\begin{aligned}
\left\langle\text { g.s. }|D| 1 \mathrm{~s}^{-1} 6 \sigma^{\prime}\right\rangle\left\langle 1 \mathrm{~s}^{-1} 6 \sigma^{\prime}|Q| 2 \mathrm{p}^{-2} 4 \mathrm{p} \sigma\right\rangle \\
\quad \times\left\langle 2 \mathrm{p}^{-2} 4 \mathrm{p} \sigma|Q| 1 \mathrm{~s}^{-1} 4 \mathrm{p} \sigma^{\prime}\right\rangle\left\langle 1 \mathrm{~s}^{-1} 4 \mathrm{p} \sigma^{\prime}|D| \text { g.s. }\right\rangle .
\end{aligned}
$$

However, $\quad\left\langle 2 \mathrm{p}^{-2} 4 \mathrm{p} \sigma|Q| 1 \mathrm{~s}^{-1} 4 \mathrm{p} \sigma^{\prime}\right\rangle\left\langle 1 \mathrm{~s}^{-1} 4 \mathrm{p} \sigma^{\prime}|D|\right.$ g.s. $\rangle=$ $\sqrt{I\left(4 \mathrm{p} \sigma^{\prime}, 4 \mathrm{p} \sigma\right)}$ cannot be obtained from the pseudo-cross section. Instead, only $\sqrt{I\left(4 \mathrm{p} \sigma^{\prime}, 4 \mathrm{p} \sigma\right)+I\left(4 \mathrm{p} \pi^{\prime}, 4 \mathrm{p} \pi\right)}=\sqrt{I\left(4 \mathrm{p}^{\prime}, 4 \mathrm{p}\right)}$ can be obtained experimentally. To take this into account, we can define $I\left(4 \mathrm{p} \sigma^{\prime}, 4 \mathrm{p} \sigma\right)=\cos ^{2}(\alpha) \cdot I\left(4 \mathrm{p}^{\prime}, 4 \mathrm{p}\right)$ and $I\left(4 \mathrm{p} \pi^{\prime}, 4 \mathrm{p} \pi\right)=\sin ^{2}(\alpha)$.
$I\left(4 \mathrm{p}^{\prime}, 4 \mathrm{p}\right)$, so that $\frac{I\left(4 \mathrm{p} \pi^{\prime}, 4 \mathrm{p} \pi\right)}{I\left(4 \mathrm{p} \sigma^{\prime}, 4 \mathrm{p} \sigma\right)}=\tan ^{2}(\alpha)$. Moreover, $\cos (\alpha)$ is identical to $\beta_{i, j}$ so that the expected fit result for the latter quantity depends on $\frac{I\left(4 \mathrm{p} \pi^{\prime}, 4 \mathrm{p} \pi\right)}{I\left(4 \mathrm{p} \sigma^{\prime}, 4 \mathrm{p} \sigma\right)}$. Based on these considerations the obtained values $\beta_{6 \sigma, 4 \mathrm{p} \sigma / \pi}=0.14$ and $\beta_{4 \mathrm{~s} \sigma, 4 \mathrm{p} \sigma / \pi}=-0.18$ result in $\frac{I\left(4 \mathrm{p} \pi^{\prime}, 4 \mathrm{p} \pi\right)}{I\left(4 \mathrm{p} \sigma^{\prime}, 4 \mathrm{p} \sigma\right)}=50$ and 30 , respectively. These values describe the decay branching ratio of $\mathrm{Cl} 1 \mathrm{~s}^{-1} 4 \mathrm{p} \pi^{\prime} / \mathrm{Cl} 1 \mathrm{~s}^{-1} 4 \mathrm{p} \sigma^{\prime}$ intermediate states to the $2 \mathrm{p}^{-2} 4 \mathrm{p}$ final state. By assuming similar total shake probabilities for the $4 \mathrm{p} \sigma$ and $4 \mathrm{p} \pi$ electrons during the Auger decay the results can be compared with different calculations for the $\mathrm{Cl}$ $1 \mathrm{~s}^{-1}$ absorption spectrum performed by Fronzoni et al. $^{33}$ These calculations result in ratios between 7.8 and 320 which correspond to values of $\beta_{i, j}$ between 0.06 and 0.34 , i.e. reasonable agreement between experiment and theory is observed. These results show that in principle, i.e. using an electron analyzer located at the magic angle with respect to the polarization axis of the light allowing measuring the absolute cross sections, our fitting procedure can allow disentangling experimentally unresolved contributions of intermediate states like $1 \mathrm{~s}^{-1} 4 \mathrm{p} \sigma^{\prime} / \pi^{\prime}$. Our measurements were done using an analyzer set parallel to polarization, which means that the presented results do not provide the absolute values. Nevertheless, we believe our results can be considered as semi-quantitative and they are coherent in comparison with the theoretical ones.

The fit analysis clearly shows that the ELI terms have to be taken into account in order to correctly describe the peak positions of the $1 \mathrm{~s}^{-1} 6 \sigma^{\prime}$ state contribution in different pseudocross sections. Moreover, the obtained values for $\beta_{i, j}$ can be interpreted semi-quantitatively, i.e. the values, which are expected to be \pm 1 , are in most cases much larger than the values, which are expected to be close to 0 . However, in more detail, some of the $\beta_{i, j}$ values differ significantly from the expected values. These differences can be explained with a number of shortcomings. Firstly, the fit model is a simplification of the reality. Secondly, the different final states cannot be completely distinguished in the 2D-map. Thirdly, the pseudocross sections can only be extracted up to a photon energy of $2828.25 \mathrm{eV}$ so that the ELI with the higher Rydberg states or even the continuum states cannot be taken into account properly. In summary, in view of the shortcomings of the data analysis, the agreement between experiment and expectation can be considered reasonable. However, in particular the latter two shortcomings can be avoided by measuring the 2D-maps with better statistics and higher resolution. Such improvements will become feasible in the near future once the planned upgrade of the GALAXIES beamline is commissioned, which will allow a more sophisticated analysis of the ELI.

Fig. 5 shows the PEY spectrum as well as the pseudo-cross sections of the final states $2 \mathrm{p}^{-2}\left({ }^{1} \mathrm{D}\right) 8 \mathrm{a}_{1}$ and $2 \mathrm{p}^{-2}\left({ }^{1} \mathrm{~S}\right) 4 \mathrm{p}$ of $\mathrm{CH}_{3} \mathrm{Cl}$ close to the $\mathrm{Cl}$ 1s ionization threshold. As for $\mathrm{HCl}$, all spectra were fitted in parallel and the black solid lines represent the fit result and the individual contributions are indicated by colored subspectra. The PEY spectrum is considerably simpler than that of $\mathrm{HCl}$ and shows only two excited states $\mathrm{Cl} 1 \mathrm{~s}^{-1} 8 \mathrm{a}_{1}{ }^{\prime}$ and $\mathrm{Cl}$ 
$1 \mathrm{~s}^{-1} 4 \mathrm{p}^{\prime}$. Nevertheless, in the fitting procedure the $\mathrm{Cl} 1 \mathrm{~s}^{-1} 5 \mathrm{p}^{\prime}$ and $\mathrm{Cl} 1 \mathrm{~s}^{-1}$ higher- $n \mathrm{p}^{\prime}$ contributions are taken into account by individual lines instead of being included in the arctangent function. This is necessary in order to describe the strong intensity decrease in the $2 \mathrm{p}^{-2}\left({ }^{1} \mathrm{~S}\right) 4 \mathrm{p}$ spectrum close to the ionization threshold, see the lower panel in Fig. 5 .

As in the case of $\mathrm{HCl}, \mathrm{ELI}$ is also present in $\mathrm{CH}_{3} \mathrm{Cl}$. It is, however, less pronounced and can mainly be observed due to the fact that the peak maximum of the $\mathrm{Cl} 1 \mathrm{~s}^{-1} 8 \mathrm{a}_{1}{ }^{\prime}$ resonance in the $2 \mathrm{p}^{-2}\left({ }^{1} \mathrm{~S}\right) 4 \mathrm{p}$ pseudo-cross section is at a slightly higher energy than in the other spectrum, see dashed vertical lines. In the fit analysis ELI was also included, although it resulted in partially ambiguous results. This can be understood mainly based on two facts. First, the rather unstructured cross section prevents an unambiguous determination of multiple fit parameters. This holds in particular for the case of the $2 p^{-2}\left({ }^{1} \mathrm{D}\right) 8 \mathrm{a}_{1}$ pseudo-cross section with practically only one spectral feature. Second, the Lorentzian lineshapes used for the Rydberg states are less accurate than in the case of $\mathrm{HCl}$ due to the different vibrational progressions. Actually, for $\mathrm{Cl} 1 \mathrm{~s}^{-1}$ core-excited or core-ionized states no vibrational progressions can be resolved due to the large lifetime broadening. However, the vibrational progression is expected to be similar to that of the $\mathrm{Cl} 2 \mathrm{p}^{-1}$ states since the exact location of the hole in such deep core shells has a negligible influence on the valence electrons responsible for the molecular geometry. The high-resolution $\mathrm{Cl} 2 \mathrm{p}^{-1}$ photoelectron spectra in $\mathrm{HCl}$ clearly show that vibrational excitations are practically absent. ${ }^{40}$ Contrary to this, highresolution $\mathrm{Cl} 2 \mathrm{p}^{-1}$ photoelectron spectra of $\mathrm{CH}_{3} \mathrm{Cl}$ show asymmetric lines with a total width of $\approx 500 \mathrm{meV}$, much broader than the experimental resolution $(\approx 85 \mathrm{meV})$ and the $\mathrm{Cl} 2 \mathrm{p}$ lifetime broadening $(\approx 100 \mathrm{meV}$ ). This strongly suggests an unresolved vibrational progression ${ }^{41}$ so that the use of a Lorentzian lineshape is not the optimal description.

Despite the described shortcomings, the fit of the $2 \mathrm{p}^{-2}\left({ }^{1} \mathrm{~S}\right) 4 \mathrm{p}$ pseudo-cross section clearly requires ELI contributions to describe the above discussed shift of the maximum of the $\mathrm{Cl} 1 \mathrm{~s}^{-1} 8 \mathrm{a}_{1}^{\prime}$ resonance and the non-negligible spectral intensity around $2825.5 \mathrm{eV}$. The red subspectrum shows the ELI contribution caused by the $\mathrm{Cl} 1 \mathrm{~s}^{-1} 8 \mathrm{a}_{1}^{\prime}$ and the $\mathrm{Cl} 1 \mathrm{~s}^{-1} 4 \mathrm{p}^{\prime}$ resonances. Depending on the exact fit model the $\beta_{8 \mathrm{a}_{1}, 4 \mathrm{p}}$ factor varies between -0.2 and -0.35 . This low $\beta_{8 \mathrm{a}_{1}, 4 \mathrm{p}}$ value indicates that the spectral structure labeled $4 \mathrm{p}$ mainly has $e$-symmetry and only little $\mathrm{a}_{1}$ one with a ratio of $\frac{I\left(4 \mathrm{pe}^{\prime}, 4 \mathrm{pe}\right)}{I\left(4 \mathrm{pa}_{1}^{\prime}, 4 \mathrm{pa}_{1}\right)}=7$ to 25 . This result is in line with the findings for $\mathrm{HCl}$ since the $4 \mathrm{pe}\left(4 \mathrm{pa}_{1}\right)$ orbital in $C_{3 \mathrm{v}}$-symmetry corresponds to the $4 \mathrm{p} \pi(4 \mathrm{p} \sigma)$ orbital in $C_{\infty \mathrm{v}}$-symmetry, i.e. the excitation into the Rydberg orbital oriented along the chemical bond is much less intense than the excitation into the Rydberg orbital perpendicular to the molecular bond.

\section{Conclusion}

In conclusion, we have recorded resonant Auger spectra in $\mathrm{HCl}$ and $\mathrm{CH}_{3} \mathrm{Cl}$ at the $\mathrm{Cl} 1$ s edge at different photon energies varied with a small step. From the obtained 2D-maps we have extracted the pseudo-cross sections for the most intense $2 \mathrm{p}^{-2}$ final states. These pseudo-cross sections allow the observation of strong shake effects in both molecules. Furthermore, the pseudo-cross sections reveal clear experimental evidence of ELI in $\mathrm{HCl}$ and $\mathrm{CH}_{3} \mathrm{Cl}$.

We have developed a fitting procedure that takes into account both the ELI effect and nuclear dynamics in dissociative core-excited states. With this fitting procedure we have utilized the ELI contributions to disentangle the unresolved states $1 \mathrm{~s}^{-1} 4 \mathrm{p} \sigma^{\prime} / \pi^{\prime}$ in $\mathrm{HCl}$ as well as $1 \mathrm{~s}^{-1} 4 \mathrm{pe}^{\prime} / \mathrm{a}_{1}{ }^{\prime}$ in $\mathrm{CH}_{3} \mathrm{Cl}$ and to extract their intensity ratios. The present measurements using an analyzer set parallel to the polarization direction of the synchrotron light are limited by the fact that an isotropic emission of the Auger electrons has to be assumed in order to compare the experimental results with theory. However, for $\mathrm{HCl}$ the comparison with theoretical values from the literature shows satisfactory agreement. Although the assumption of an isotropic Auger emission is supported by the agreement between experiment and theory, it is obvious that for more accurate future measurements the analyzer has to be set to the magic angle.

In summary, we are convinced that ELI is a general phenomenon in molecules excited at deep core levels, where large lifetime broadening allows coherent excitation of different intermediate states. The results presented in this work suggest that ELI can be used to obtain more detailed information about the excited states where deep core holes are involved.

\section{Acknowledgements}

Experiments were performed on the GALAXIES beamline at SOLEIL Synchrotron, France (Proposals No. 20120122 and 20130309). We are grateful to D. Prieur for technical assistance and to SOLEIL staff for smoothly running the facility.

\section{References}

1 P. Morin and I. Nenner, Phys. Rev. Lett., 1986, 56, 1913.

2 H. Aksela, S. Aksela, M. Ala-Korpela, O.-P. Sairanen, M. Hotokka, G. M. Bancroft, K. H. Tan and J. Tulkki, Phys. Rev. A: At., Mol., Opt. Phys., 1990, 41, 6000.

3 A. Menzel, B. Langer, J. Viefhaus, S. B. Whitfield and U. Becker, Chem. Phys. Lett., 1996, 258, 265.

4 O. Björneholm, S. Sundin, R. R. T. Marinho, A. Naves de Brito, F. Gel'mukhanov and H. Ågren, Phys. Rev. Lett., 1997, 79, 3150.

5 R. Feifel, F. Burmeister, P. Sałek, M. N. Piancastelli, M. Bässler, S. L. Sorensen, C. Miron, H. Wang, I. Hjelte, O. Björneholm, A. Naves de Brito, F. Gel'mukhanov, H. Ågren and S. Svensson, Phys. Rev. Lett., 2000, 85, 3133.

6 E. Kukk, A. Wills, N. Berrah, B. Langer, J. D. Bozek, O. Nayadin, M. Alsherhi, A. Farhat and D. Cubaynes, Phys. Rev. A: At., Mol., Opt. Phys., 1998, 57, 1485.

7 E. Sokell, A. A. Wills, M. Weidenhoeft, X. Feng, D. Rolles and N. Berrah, J. Phys.: Conf. Ser., 2007, 88, 012007.

8 I. Hjelte, M. N. Piancastelli, R. F. Fink, O. Björneholm, M. Bässler, R. Feifel, A. Giertz, H. Wang, K. Wiesner, 
A. Ausmees, C. Miron, S. L. Sorensen and S. Svensson, Chem. Phys. Lett., 2001, 334, 151.

9 O. Björneholm, M. Bässler, A. Ausmees, I. Hjelte, R. Feifel, H. Wang, C. Miron, M. N. Piancastelli, S. Svensson, S. L. Sorensen, F. Gel'mukhanov and H. Ågren, Phys. Rev. Lett., 2000, 84, 2826.

10 I. Hjelte, M. N. Piancastelli, C. M. Jansson, K. Wiesner, O. Björneholm, M. Bässler, S. L. Sorensen and S. Svensson, Chem. Phys. Lett., 2003, 370, 781.

11 F. Gel'mukhanov and H. Ågren, Phys. Rep., 1999, 312, 87.

12 M. Simon, L. Journel, R. Guillemin, W. C. Stolte, I. Minkov, F. Gel'mukhanov, P. Sałek, H. Ågren, S. Carniato, R. Taïeb, A. C. Hudson and D. W. Lindle, Phys. Rev. A: At., Mol., Opt. Phys., 2006, 73, 020706.

13 T. Marchenko, L. Journel, T. Marin, R. Guillemin, S. Carniato, M. Žitnik, M. Kavčič, K. Bučar, A. Mihelič, J. Hoszowska, W. Cao and M. Simon, J. Chem. Phys., 2011, 134, 144308.

14 M. N. Piancastelli, G. Goldsztejn, T. Marchenko, R. Guillemin, R. K. Kushawaha, L. Journel, S. Carniato, J.-P. Rueff, D. Céolin and M. Simon, J. Phys. B: At., Mol. Opt. Phys., 2014, 47, 124031.

15 M. Žitnik, M. Kavčič, R. Bohinc, K. Bučar, A. Mihelič, W. Cao, R. Guillemin, L. Journel, T. Marchenko, S. Carniato, E. Kawerk, M. N. Piancastelli and M. Simon, J. Electron Spectrosc. Relat. Phenom., 2015, 204, 356-364.

16 U. Fano, Phys. Rev., 1961, 124, 1866.

17 T. Åberg, Phys. Scr., 1992, T41, 71.

18 A. Cesar and H. Ågren, Phys. Rev. A: At., Mol., Opt. Phys., 1992, 45, 2833.

19 J. C. Levin, C. Biedermann, N. Keller, L. Liljeby, C.-S. O. R. T. Short, D. W. Lindle and I. A. Sellin, Phys. Rev. Lett., 1990, 65, 8.

20 J.-E. Rubensson, M. Neeb, A. Bringer, M. Biermann and W. Eberhardt, Chem. Phys. Lett., 1996, 257, 447.

21 T. LeBrun, S. H. Southworth, G. B. Armen, M. A. MacDonald and Y. Azuma, Phys. Rev. A: At., Mol., Opt. Phys., 1999, 60, 6.

22 D. Céolin, T. Marchenko, R. Guillemin, L. Journel, R. K. Kushawaha, S. Carniato, S.-M. Huttula, J. P. Rueff, G. B. Armen, M. N. Piancastelli and M. Simon, Phys. Rev. A: At., Mol., Opt. Phys., 2015, 91, 022502.

23 G. B. Armen, J. C. Levin and I. A. Sellin, Phys. Rev. A: At., Mol., Opt. Phys., 1996, 53, 772.

24 R. K. Kushawaha, K. Jänkälä, T. Marchenko, G. Goldsztejn, R. Guillemin, L. Journel, D. Céolin, J.-P. Rueff, A. F. Lago, R. Püttner, M. N. Piancastelli and M. Simon, Phys. Rev. A: At., Mol., Opt. Phys., 2015, 92, 013427.

25 M. Kavčič, M. Žitnik, K. Bučar, A. Mihelič, S. Carniato, L. Journel, R. Guillemin and M. Simon, Phys. Rev. Lett., 2010, 105, 113004.
26 J.-I. Adachi, N. Kosugi and A. Yagishita, J. Phys. B: At., Mol. Opt. Phys., 2005, 38, R127.

27 J.-P. Rueff, J. M. Ablett, D. Céolin, D. Prieur, T. Moreno, V. Balédent, B. Lassalle, J. E. Rault, M. Simon and A. Shukla, J. Synchrotron Radiat., 2015, 22, 175.

28 D. Céolin, J. M. Ablett, D. Prieur, T. Moreno, J.-P. Rueff, B. Pilette, T. Marchenko, L. Journel, T. Marin, R. Guillemin and M. Simon, J. Electron Spectrosc. Relat. Phenom., 2013, 190, 188.

29 M. Simon, R. Püttner, T. Marchenko, R. Guillemin, R. K. Kushawaha, L. Journel, G. Goldsztejn, M. N. Piancastelli, J. M. Ablett, J.-P. Rueff and D. Céolin, Nat. Commun., 2014, 5, 4069.

30 R. Püttner, G. Goldsztejn, D. Céolin, J.-P. Rueff, T. Moreno, R. K. Kushawaha, T. Marchenko, R. Guillemin, L. Journel, D. W. Lindle, M. N. Piancastelli and M. Simon, Phys. Rev. Lett., 2015, 114, 193001.

31 V. Schmidt, Electron Spectroscopy of Atoms using Synchrtron Radiation, Cambridge University Press, 1997, ISBN 0-52155053-X.

32 R. Guillemin, S. Sheinerman, R. Püttner, T. Marchenko, G. Goldsztejn, L. Journel, R. K. Kushawaha, D. Céolin, M. N. Piancastelli and M. Simon, Phys. Rev. A: At., Mol., Opt. Phys., 2015, 92, 012503.

33 G. Fronzoni, M. Stener, P. Decleva and G. De Alti, Chem. Phys., 1998, 232, 9.

34 R. Püttner, V. Pennanen, T. Matila, A. Kivimäki, M. Jurvansuu, H. Aksela and S. Aksela, Phys. Rev. A: At., Mol., Opt. Phys., 2002, 65, 042505.

35 M. Poygin, R. Püttner, M. Martins, V. Pennanen, M. Jurvansuu, Y. Jiang, H. Aksela, S. Aksela and G. Kaindl, Phys. Rev. A: At., Mol., Opt. Phys., 2006, 74, 012711.

36 R. Püttner, Y. F. Hu, G. M. Bancroft, H. Aksela, E. Nõmmiste, J. Karvonen, A. Kivimäki and S. Aksela, Phys. Rev. A: At., Mol., Opt. Phys., 1999, 59, 4438.

37 P. Sałek, F. Gel'mukhanov and H. Ågren, Phys. Rev. A: At., Mol., Opt. Phys., 1999, 59, 1147-1159.

38 J. L. Campbell and T. Papp, At. Data Nucl. Data Tables, 2001, $77,1$.

39 E. Kukk, H. Aksela, A. Kivimäki, J. Jauhiainen, E. Nõmmiste and S. Aksela, Phys. Rev. A: At., Mol., Opt. Phys., 1997, 56, 1481.

40 M. Kivilompolo, A. Kivimäki, M. Jurvansuu, H. Aksela, S. Aksela and R. F. Fink, J. Phys. B: At., Mol. Opt. Phys., 2000, 33, L157.

41 A. Rosso, A. Lindblad, M. Lundwall, T. Rander, S. Svensson, M. Tchaplyguine, G. Öhrwall and O. Björneholm, J. Chem. Phys., 2007, 127, 024302. 